\title{
THE LINEAR CONGRUENCE GROUP MODULO $n$
}

\author{
F. A. LEWIS
}

The symbol $G L H[m, n]$ will be used to represent the order of $G L H(m, n)$, the group of linear transformations on $m$ variables whose coefficients are taken modulo $n$ in such a way that the determinant of each transformation is prime to $n$. In this note we state four theorems on congruence groups, which may be obtained by modifying proofs of corresponding theorems ${ }^{1}$ on groups of transformations with coefficients in a Galois field $G F\left[p^{n}\right]$. Theorem 5 gives a set of defining relations for a related abstract group.

ThEOREM 1. $G L H[m, n]=\prod_{i=1}^{m} n^{i-1} \phi_{i}(n)$, where $\phi_{i}(n)$ represents the ith totient of $n$.

THEOREM 2. The matrix of every transformation of $G L H(m, n)$ of determinant s equals $B D_{s}$, where $B$ is derived from $B_{r, c, \lambda}$ and $D_{s}$ is the diagonal matrix $(1,1, \cdots, s)$.

TheOREM 3. $S L H[m, n]=G L H[m, n] / \phi(n)$.

CoROLlary. $S L H[2, n]=n \phi_{2}(n)$.

THEOREM 4. $S L H(2, n)=\{V, W\}$, where $V$ and $W$ are, respectively, the following transformations: $x_{1}^{\prime}=-x_{2}, x_{2}^{\prime}=x_{1}$ and $x_{1}^{\prime}=x_{1}, x_{2}^{\prime}=x_{1}+x_{2}$.

THEOREM $^{2}$ 5. If $n>2, \operatorname{SLH}(2, n)$ is simply isomorphic with the abstract group whose generators $V$ and $W$ satisfy

(a) $V^{4}=I$,

(b) $W^{n}=I, W V^{2}=V^{2} W$,

(c) $W^{\lambda} V W^{\mu} V W^{(\lambda+1) /(\lambda \mu-1)} V W^{\lambda \mu-1} V W^{(\mu+1) /(\lambda \mu-1)} V=I$, for all values of $\lambda$ and $\mu$ such that $\lambda \mu-1$ is prime to $n$.

Let $g$ be the order of $G=\{V, W\}$. Since (a), (b), and (c) are satisfied by the generators of $S L H(2, n), g \geqq n \phi_{2}(n)$.

If $\mu$ is prime to $n$, the substitutions $\lambda=\alpha(1+1 / \beta)$ and $\mu=1 / \alpha$ in (c) yield

(c') $W^{\alpha+\alpha / \beta} V W^{1 / \alpha} V W^{\alpha+\alpha \beta+\beta} V W^{1 / \beta} V W^{\beta+\beta / \alpha} V=I$, for all $\alpha$ and $\beta$ prime to $n$.

In order to simplify the computation, we define

Received by the editors August 4, 1950 and, in revised form, August 9, 1951.

${ }^{1}$ See Dickson, Linear groups, pp. 77-82, for statement of corresponding theorems and explanation of notation.

${ }^{2}$ The corresponding theorem on $\operatorname{SLH}\left(2, p^{n}\right)$ is due to E. H. Moore; Dickson, loc. cit., p. 300. 


$$
R_{\alpha}=W^{1 / \alpha} V W^{\alpha} V W^{1 / \alpha} V
$$

for all values of $\alpha$ prime to $n$. The following properties of the operator $R$ may be established:

(d) $R_{1}=I$,

(e) $\left(R_{\alpha} V\right)^{2}=V^{2}$,

(f) $W^{\rho} R_{\alpha}=R_{\alpha} W^{\rho \alpha^{2}}$, where $\alpha$ is prime to $n$ and $\rho$ is arbitrary.

(f') $R_{\alpha} V=V R_{1 / \alpha}$,

(g) $R_{\alpha \beta}=R_{\alpha} R_{\beta}$.

Consider the following set of elements

$$
W^{(c+d x) /(a+b x)} R_{a+b x} V^{-1} W^{-b /(a+b x)} V W^{-x},
$$

where $(a, b)$ is prime to $n, x$ is any integer such that $a+b x$ is prime to $n$, and $a d-b c \equiv 1(\bmod n)$. The condition

$$
\begin{aligned}
W^{(c+d x) /(a+b x)} R_{a+b x} V^{-1} W^{-b /(a+b x)} V W^{-x} & \\
& =W^{(c+d y) /(a+b y)} R_{a+b y} V^{-1} W^{-b /(a+b y)} V W^{-y}
\end{aligned}
$$

for all values of $x$ and $y$ for which $a+b x$ and $a+b y$ are prime to $n$ reduces to an equivalent form of (c). Hence a different choice of $x$ yields the same set $(h)$. Therefore, the number of distinct elements in the set is at most $n \phi_{2}(n)$.

It we multiply the set on the right by $W$, the product has the same form as (h). Applying $V$ as a right-hand multiplier, the product of any element of the set by $V$ is an element of the set if

$$
\begin{aligned}
W^{(c+d x) /(a+b x)} R_{a+b x} V^{-1} W^{-b /(a+b x)} & V W^{-x} V \\
& =W^{(d-c y) /(b-a y)} R_{b-a y} V^{-1} W^{a /(b-a y)} V W^{-y},
\end{aligned}
$$

where $b-a y$ is prime to $n$. This condition may be reduced to $\left(c^{\prime}\right)$ by means of (c) and the fact that $x$ and $y$ may be chosen so that $a+b x, b-a y$, and $1+x y$ are each relatively prime to $n$. Hence $g$ $=n \phi_{2}(n)$ and the theorem is proved.

The University of Alabama 